\title{
Suspensão de cirurgia de catarata e suas
}

\section{causas}

\section{Reasons for cataract surgery cancelation}

\author{
Rodrigo Pessoa Cavalcanti Lira, Maurício Abujamra Nascimento, Edméa Rita \\ Temporini, Newton Kara-José e Carlos Eduardo Leite Arieta
}

Departamento de Oftalmo-Otorrinolaringologia. Universidade Estadual de Campinas. Campinas, SP, Brasil

\section{Descritores}

Extração de catarata. ${ }^{\#}$ Hospitais universitários." - Causas de suspensão.

Keywords

Cataract extraction. ${ }^{\#}$ Hospitals, university. ${ }^{*}$ - Causes of suspension.

\begin{abstract}
Resumo
Com o objetivo de verificar as causas de suspensão de cirurgia de catarata e sugerir medidas para melhorar a eficiência do serviço prestado à população, foi realizado um estudo transversal no serviço de oftalmologia de um hospital universitário do Estado de São Paulo. A amostra foi composta por 200 indivíduos. A média de idade foi de $68 \pm 11,4$ anos. As causas de suspensão de cirurgia foram: condição clínica desfavorável (23,1\%); horário cirúrgico insuficiente (35,9\%); e não comparecimento do paciente $(41 \%)$. Os fatores causadores foram, majoritariamente, as razões sociais e o funcionamento do hospital.
\end{abstract}

\begin{abstract}
To study the reasons for canceling cataract surgeries, and to suggest actions to improve the efficiency of patient care. A cross-sectional study was carried out in a university hospital's ophthalmology clinic of the state of São Paulo, Brazil. Two hundred subjects were randomly selected. The mean age was $68 \pm 11.4$ years old. The reasons for canceling surgery were: unpropitious clinical condition (23.1\%); tight schedule (35.9\%); and patient non-attendance (41\%). Most of the reasons related to social issues and the hospital's administrative aspects.
\end{abstract}

A catarata é a maior causa de cegueira no mundo, atingindo em torno de 16.000 .000 de pessoas. ${ }^{5}$ Estudos indicam que $12 \%$ a $50 \%$ de pessoas acima de 65 anos têm catarata. ${ }^{5}$ Esse quadro é passível de reversão por intervenção cirúrgica apropriada, sendo a facectomia um procedimento feito geralmente com anestesia local, eficaz, de baixo custo e praticado com segurança há vários anos. ${ }^{3}$

Levando-se em consideração que o Brasil teve uma meta de realização de 262.000 cirurgias de catarata no ano de 2.000 - parte de uma programação que visa acabar com a cegueira endêmica por essa afec- ção até $2002^{4}$-, o desperdício de horário cirúrgico com o cancelamento de intervenções por motivos atenuáveis deve ser combatido mediante um planejamento eficiente.

A presente pesquisa objetiva verificar as causas de suspensão de cirurgia de catarata em um hospital universitário, a fim de sugerir medidas para melhorar a eficiência do serviço prestado à população.

\section{MÉTODOS}

Foi realizado um estudo transversal em um hospi-
Correspondência para/Correspondence to: Rodrigo Pessoa Cavalcanti Lira

Rua Visconde de Itaparica, 174, apto 203 B

50710-090 Recife, PE, Brasil

E-mail: rpclira@uol.com.br
Apresentado no $14^{\circ}$ Congresso Brasileiro de Prevenção da Cegueira e Reabilitação Visual, Natal, RN, 2000 Recebido em 6/12/2000. Reapresentado em 8/3/2001. Aprovado em 1/8/2001. 
tal universitário do Estado de São Paulo, Brasil, que realiza cerca de 3.000 facectomias anualmente.

A amostra foi obtida por sorteio de pacientes adultos (com idade igual ou superior a 40 anos) com indicação de cirurgia de catarata com implante de lente intra-ocular sob anestesia local no ambulatório.

Para caracterizar a amostra, foram selecionadas as variáveis "sexo" e "idade". Os dados foram obtidos em ficha de registro do paciente, referente ao primeiro semestre do ano de 2000.

Para o agendamento da cirurgia, todos os pacientes participaram de um grupo de orientação pré-operatório e submeteram-se a exames de hemoglobina, glicemia de jejum e eletrocardiograma, além de avaliação clínica, conforme a rotina do serviço.

As causas de suspensão de cirurgia foram classificadas em: administrativas; não-comparecimento do paciente; e condições clínicas inadequadas. As administrativas incluíram: falta de prontuário; ausência de funcionários; falta de horário; e falta de material cirúrgico.

\section{RESULTADOS}

A amostra foi composta por 200 indivíduos de ambos os sexos (53,5\% homens e 46,5\% mulheres). A média de idade foi de 68 anos (desvio-padrão de 11,4 anos), não havendo diferença significativa entre a idade média dos pacientes, de acordo com a realização ou suspensão de cirurgia (68,02 anos nos pacientes com cirurgia realizada e 67,94 anos nos casos de suspensão $-\mathrm{p}=0,916$ ).

Foram suspensas 39 cirurgias (19,5\%), cujas causas não relacionadas à condição clínica do paciente representaram 77\% (Tabela).

\section{DISCUSSÃO}

Nos países em desenvolvimento, é crescente o estudo de tecnologias de baixo custo que possam ser úteis no aproveitamento de recursos limitados. ${ }^{1,5} \mathrm{~A}$ utilização máxima da capacidade cirúrgica constitui

\begin{tabular}{lcrr}
\multicolumn{4}{l}{ Tabela - Causas de suspensão de cirurgia de catarata. } \\
\hline Causa & Freqüência & $\begin{array}{r}\text { \% cirurgia } \\
\text { suspensas }\end{array}$ & $\begin{array}{r}\% \text { total de } \\
\text { cirurgias }\end{array}$ \\
\hline Não-comparecimento & 16 & 41,0 & 8,0 \\
Horário cirúrgico & 14 & 35,9 & 7,0 \\
Condição clínica & 9 & 23,1 & 4,5 \\
\hline Total & 39 & 100,0 & 19,5 \\
\hline
\end{tabular}

uma das principais medidas que visam à eficiência do uso de verbas em uma unidade hospitalar. ${ }^{1} \mathrm{Na}$ área de oftalmologia, no Brasil, são raros os relatos científicos a respeito da produtividade de centros cirúrgicos ou, mais especificamente, em relação às cirurgias de catarata. ${ }^{1,2}$

No presente estudo, houve um índice de suspensão de cirurgia de catarata de $19,5 \%$. Arieta et al ${ }^{1}$ avaliaram o comportamento das cirurgias ambulatoriais oftalmológicas, realizadas no mesmo hospital universitário, em 1992, e detectaram 22,8\% de casos de suspensões. Esses resultados sugerem que, apesar de esforços no sentido de melhorar o funcionamento desse centro cirúrgico ambulatorial nos últimos anos, ${ }^{1,2}$ ainda persiste elevada fração de cirurgias suspensas.

Causas relacionadas à condição clínica do paciente levaram à suspensão de 4,5\% das cirurgias, contrastando com a situação desse mesmo serviço há oito anos, quando tais eventos foram responsáveis pelo cancelamento de 12,6\% das cirurgias. ${ }^{1}$ Esse fato se deve, provavelmente, à melhor avaliação clínica do paciente cirúrgico, em consequiência da implantação de um grupo de orientação pré-operatória no serviço de oftalmologia, em 1993.

Os casos de suspensão de cirurgia por limitação de vagas em razão de horário disponível aumentaram de $2,5 \%$ para $7 \%$, em $1992,{ }^{1}$ no presente estudo, evidenciando a necessidade de dimensionar a capacidade resolutiva do centro cirúrgico ambulatorial às reais necessidades do serviço de oftalmologia. Ações administrativas, no sentido de otimizar o funcionamento de um centro cirúrgico ambulatorial, podem levar a um aumento de até $36 \%$ no número de cirurgias de catarata sem haver ônus adicional dos custos operacionais. ${ }^{2}$

A proporção de cirurgias suspensas devido ao nãocomparecimento do paciente foi a mesma de oito anos atrás. ${ }^{1}$ Não se dispõe de estudo específico sobre as causas de não-comparecimento. A dificuldade econômica do paciente para custear o seu transporte e do acompanhante foi responsável por mais de $30 \%$ da não-procura de tratamento, de acordo com estudos prévios. ${ }^{1-3}$

Os resultados observados mostram que é preciso atenuar os fatores causadores da suspensão de cirurgias de catarata que, na maioria, não parecem estar relacionados à condição clínica do paciente. Possivelmente, são passíveis de ser solucionados ou minimizados, com conseqüente melhor aproveitamento dos recursos disponíveis. 


\section{REFERÊNCIAS}

1. Arieta CEL, Taiar A, Kara-José N. Utilização e causas de suspensão de intervenções cirúrgicas oculares em centro cirúrgico ambulatorial universitário. Rev Assoc Med Bras 1995;41:233-5.

2. Arieta CEL, Kara-José N, Carvalho-Filho DM, Alves MR. Optimisation of a cataract-patient care service in Campinas, Brazil. Ophthalmic Epidemiol 1999;6:1-11.
3. Kara-José N, Temporini ER. Cirurgia de catarata: o porquê dos excluídos. Rev Panam Salud Publica 1999;6:242-8.

4. Monteiro JV. Campanha nacional de cirurgias de catarata 2000. J Oftalmol Jota Zero 2000;74:4-5.

5. Schwab L. Cataract. In: Schwab L. Eye care in developing nations. 3rd ed. San Francisco: The Foundation of the American Academy of Ophthalmology; 1999. p. 21-52. 